Musées, Patrimoine et Culture scientifiques et techniques

$157 \mid 2015$

janvier-février 2015

\title{
Actualité récente et évolution des pratiques autour des restes humains patrimonialisés
}

Laure Cadot

\section{OpenEdition}

\section{Journals}

Édition électronique

URL : http://journals.openedition.org/ocim/1482

DOI : 10.4000/ocim.1482

ISSN : 2108-646X

Éditeur

OCIM

Édition imprimée

Date de publication : 1 janvier 2015

Pagination : $32-34$

ISSN : 0994-1908

Référence électronique

Laure Cadot, « Actualité récente et évolution des pratiques autour des restes humains

patrimonialisés », La Lettre de l'OCIM [En ligne], 157 | 2015, mis en ligne le 01 janvier 2016, consulté le 10 décembre 2020. URL : http://journals.openedition.org/ocim/1482 ; DOI : https://doi.org/10.4000/ ocim. 1482

Ce document a été généré automatiquement le 10 décembre 2020.

Tous droits réservés 


\section{Actualité récente et évolution des pratiques autour des restes humains patrimonialisés}

\section{Laure Cadot}

\section{OCy M}

Les restes humains :

une gageure pour les musées?

\section{Laure Cadot *}

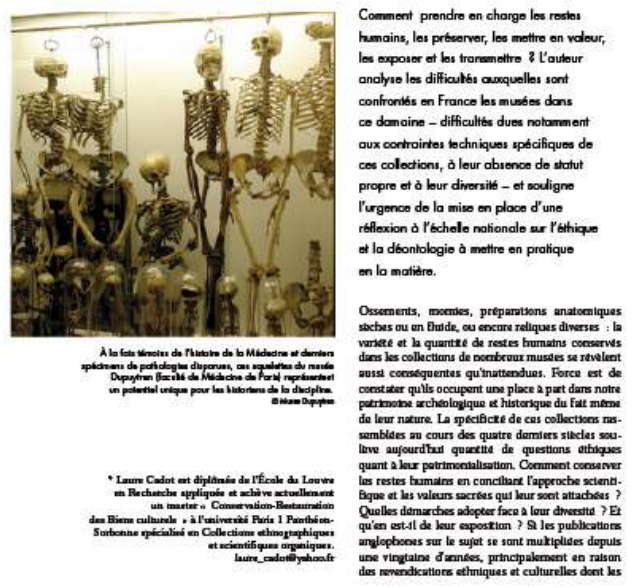

L'article (disponible sur www.ocim.fr, rubrique La Lettre de l'OCIM) soulignait l'urgence à prendre en charge les restes humains, les préserver, les mettre en valeur, les exposer et les transmettre.

Huit ans après la parution d'un précédent article dans cette même revue, la problématique des restes humains dans les collections a, bon gré mal gré, fait son chemin 
au sein des institutions muséales. Il est vrai que le sujet a défrayé la chronique à maintes reprises ces dernières années, notamment au travers de deux affaires emblématiques qui ont pour le moins contribué à alimenter et enrichir le débat autour de la légitimité de la présence des restes humains dans les collections patrimoniales et des conditions de leur exposition. On pense bien sûr, pour le premier aspect, au long et chaotique processus de restitution des têtes maories par la France à la Nouvelle-Zélande. Il aura fallu attendre cinq ans entre le vote en 2007 de la restitution de la tête conservée au muséum de Rouen par le conseil municipal de la ville, point de départ de la procédure juridique, et la cérémonie de restitution de vingt têtes issues de onze institutions françaises qui s'est déroulée en janvier 2012 au musée du Quai Branly. Au cœur du débat, la manière d'envisager les demandes de restitution concernant des restes humains qualifiés de "sensibles" sans mettre à mal le principe d'inaliénabilité des collections garant de leur pérennité. Point de départ d'une crispation forte entre les partisans du respect unilatéral $\mathrm{du}$ droit des peuples autochtones et les tenants du caractère universel des collections, la remise en cause de la décision du conseil municipal de la Ville de Rouen par le ministère de la Culture s'est en définitive appuyée sur un vice de procédure, en l'occurrence l'absence de consultation de la Commission nationale de déclassement prévue par la loi "musée" de 2002 (article 16.3 du décret $n^{\circ}$ 2002-628 du 25 avril 2002) mais néanmoins jamais constituée depuis lors. Cette affaire, sur les détails de laquelle nous ne nous attarderons pas, aura eu deux conséquences juridiques principales: la promulgation d'une loi spécifique pour la restitution des têtes maories, manière de clore le débat sur cette question spécifique sans statuer de manière générale sur le cas des restes humains dans leur ensemble et l'écartement par le législateur de la légitimité de l'article 16-1 du Code civil ${ }^{(2)}$ appliqué aux restes humains patrimoniaux et mis en avant par la municipalité de Rouen: "Le corps humain, ses éléments et ses produits ne peuvent faire l'objet d'un droit patrimonial". Ce deuxième aspect qui semble désormais faire jurisprudence, contribue à la reconnaissance de la valeur scientifique de ces collections en les plaçant prioritairement sous le régime de protection des biens culturels bénéficiant du label "Musée de France".

$2 \mathrm{Au}$ printemps 2009, alors même que la proposition de loi pour la restitution des têtes maories est à l'étude au parlement, c'est une autre affaire qui vient remettre sur le devant de la scène la problématique des restes humains patrimonialisés, mais cette fois sous l'angle de leur exposition. Nous voulons bien sûr parler de la fermeture sur décision de justice de l'exposition itinérante de cadavres plastinés Our body: à corps ouvert, présentée à Marseille puis Lyon avant d'arriver à Paris en février 2009. Sans que cette affaire touche directement les musées puisqu'il s'agit d'une manifestation organisée par une société d'évènementiel à but lucratif, les débats qui se sont focalisés sur la décence et le caractère pédagogique discutable d'une telle présentation ont néanmoins jeté un temps le discrédit sur les collections anatomiques historiques par un effet d'amalgame et de décontextualisation de certains propos. Cet épisode a toutefois permis d'enrichir à sa manière la réflexion sur les aspects éthiques et déontologiques, formalisés notamment par un avis du Comité Consultatif National d'Éthique saisi pour l'occasion'.

Ces deux cas ont eu le mérite de poser des questions sensibles de manière parfois abrupte avec leur lot inévitable de déformation et de raccourci véhiculés par les médias, mais ont amorcé une volonté de discussion et d'échange au sein de la profession de manière plus ouverte. Afin de ne pas réduire la question de la restitution des têtes maories à son seul aspect juridique et de calmer l'agitation médiatique autour des échauffements entre les différents partis, un symposium international ${ }^{2}$ a ainsi été organisé dans l'urgence au 
musée du Quai Branly à la demande du ministère de la Culture afin de débattre des questions éthiques autour du sujet plus général des restes humains. Ce symposium aura permis d'initier une réflexion d'envergure au sein de la profession donnant lieu à l'organisation de plus en plus fréquente de colloques et tables rondes interdisciplinaires où les questions de déontologie côtoient l'histoire des collections ainsi que des aspects plus pragmatiques sur la conservation, l'étude et l'exposition des restes humains dans leur diversité. Conservateurs, juristes, médecins, historiens, archéologues, anthropologues, amorcent ainsi depuis quelques années un dialogue qui permet progressivement de rompre l'isolement de chaque discipline sur ce sujet transversal. Journée d'étude consacrée à La conservation des collections ostéologiques et des restes humains dans les musées à Caen en 2008 (organisé par le musée de Normandie en collaboration avec le Mémorial de Caen), Rencontre autour du cadavre à Marseille en 2010 (organisée par le Groupe d'anthropologie et d'archéologie funéraire), colloque Les collections anatomiques: de la connaissance à la mise en valeur en 2012 (organisé par l'INP et l'université Montpellier 1) et j'en passe... ces réunions d'envergure trouvent un écho dans la mise en place de séminaires d'enseignement à l'Institut National du Patrimoine - qui a intégré dans le cursus de formation des élèves conservateur une séance de sensibilisation à la conservation des restes humains depuis 2008 - , à l'École du Louvre ou à l'EHESS. La multiplication ces dernières années des mémoires de recherche et travaux d'étudiants sur les problématiques récurrentes de l'exposition, du statut juridique ou de la préservation des restes humains participe également à la dynamisation de ce domaine en pleine effervescence.

4 Le fruit de ces discussions encore récentes en France mais maintenant amorcées de longue date à l'échelle internationale participe désormais à l'orientation et à la standardisation des pratiques professionnelles. Loin d'être considéré comme un point final, les paragraphes introduits dans Le Code de Déontologie de l'ICOM en 2004 (paragraphes $2.5 ; 3.7 ; 4.3)$ sont encore aujourd'hui précisés et affinés à la lumière de l'actualité du domaine. Le groupe "Histoire naturelle" de l'ICOM a notamment introduit en 2013 un premier chapitre approfondissant la question des restes humains, et notamment celle des restitutions, à l'occasion de la révision de sa charte.

5 En France, l'évolution la plus significative des textes et recommandations d'ordre professionnel concerne le marché de l'art puisqu'après avoir été maintes fois pointé du doigts pour son absence de prise de position, le conseil des ventes volontaires de meubles aux enchères publiques a ajouté une note relative aux restes humains dans son recueil des obligations déontologiques paru en février 2012 (paragraphe 1.5.4 Qualité des objets) qui stipule que "sauf lorsqu'ils constituent sans équivoque des biens culturels, l'opérateur de ventes volontaires s'abstient de présenter à la vente tout ou partie de corps ou de restes humains ou tout objet composéa à partir de corps ou de restes humains".

6 Sur le terrain, ces évolutions se traduisent par une prise de conscience globale des professionnels en charge des collections qui va dans le sens d'une amélioration des standards de conservation au quotidien. Conscientes des enjeux éthiques, déontologiques et scientifiques qui entourent ces collections, des institutions muséales de toute taille ont multiplié les études de ces restes notamment par le biais de l'imagerie médicale et des observations menées par des équipes pluridisciplinaires rassemblées à ces occasions. Pour les momies égyptiennes, on peut ainsi mentionner le projet pionnier par son ampleur mené par le Service du Récolement et des Dépôts du musée du Louvre en 2011 sur une quarantaine de corps desséchés de la nécropole d'Antinoé réparti dans une dizaine de 
musées ${ }^{3}$. Des études plus ponctuelles sont également conduites dans la perspective d'expositions ou tout simplement de l'approfondissement des connaissances qui sont ensuite diffusées auprès du public et des professionnels via des expositions-dossiers (après un partage sur les réseaux sociaux de l'opération de scanner de ses momies, le muséum de Lille a notamment présenté les résultats de l'étude lors de la Fête de la Science en octobre 2014), des publications ou des réunions de groupes de recherche comme celles du GDR 3446 "Centre d'Investigation et de recherche sur les momies (CIRM)" rattaché au CNRS et mis en place ces dernières années.

7 L'attention renouvelée pour ces collections, que ce soit au travers de leur étude ou de leur présentation au public, s'accompagne désormais d'un questionnement quasi systématique sur la qualité des conditions de conservation et les opérations à mettre en œuvre en terme de conditionnement, transport, manipulation, contrôle environnemental afin de garantir un niveau d'exigence minimal dans tous ces aspects. Ces considérations conservatoires semblent désormais faire partie intégrante de la démarche de respect recommandée par les textes, au même titre que le questionnement sur le caractère présentable de tel ou tel spécimen et la justification de son exposition. Les réalisations récentes et à venir du musée des Confluences et du musée de l'Homme tendent à illustrer ces réflexions collégiales alliant projet scientifique, conservation, et muséographie.

8 À la lumière de l'actualité et des réalisations de ces dernières années, on peut dire que la question des restes humains patrimonialisés, jusqu'alors assez confidentielle, est apparue sur le devant de la scène de manière un peu subite mais néanmoins salutaire. L' "effet de mode" que l'on pouvait soupçonner un moment ne s'est pas départi et une fois passée la tourmente des grands dossiers médiatiques, on n'a pu que constater le renouveau de la prise de conscience professionnelle. C'est peut être la rupture de l'isolement du fait du débat public qui aura le plus marqué ces dernières années. Pour autant, beaucoup de travail et de sensibilisation restent à accomplir et la dynamique amorcée nécessite le maintien d'une réflexion permanente sur les pratiques et les retours d'expérience afin de poursuivre la dédramatisation des enjeux de la conservation des restes humains en les clarifiant et en améliorant les ressources disponibles afin d'optimiser le travail au quotidien sur ces collections.

\section{NOTES}

1. Comité Consultatif National d'Éthique pour les Sciences de la Vie et de la Santé (Rapporteurs : Jean-Claude Ameisen et Pierre Le Coz), Avis $n^{\circ} 111$ sur les problèmes éthiques posés par l'utilisation des cadavres à des fins de conservation ou d'exposition muséale, 7 janvier 2010. Disponible en ligne : www.ccne-ethique.fr/sites/default/files/publications/avis_111.pdf

2. Des collections anatomiques aux objets de culte: conservation et exposition des restes humains dans les musées, 22 et 23 février 2008, transcription disponible en ligne : www.quaibranly.fr/fileadmin/user_upload/pdf/

Version_Francaise_Symposium_Restes_Humains.pdf

3. Antinoé : Momies, textiles, céramiques et autres antiques, sous la direction de Yannick Lintz et Magali Coudert, Louvre-Somogy, 2014. 


\section{RÉSUMÉS}

À l'occasion des 30 ans de l'OCIM, La Lettre de l'OCIM propose à ses lecteurs, dans chaque numéro de l'année 2015, un retour sur un article marquant de l'histoire de la revue. À partir d'un corpus d'articles choisis par la rédaction de La Lettre de l'OCIM, les membres du comité des Publications de l'OCIM ont sélectionné plusieurs contributions.

Dans cette perspective, il a été demandé à l'auteur ou à un expert du domaine de revisiter la problématique exposée dans l'article à la lueur des changements intervenus - notamment dans les pratiques professionnelles - depuis son écriture et de proposer des éléments prospectifs sur la question.

Pour ce numéro de janvier-février 2015, Laure Cadot spécialiste en conservation-restauration d'objets ethnographiques (matériaux organiques, polychromies mates et restes humains) revient sur son article "Les restes humains : une gageure pour les musées ?" publié dans le n 109 de La Lettre de l'OCIM en janvier-février 2007 et qui analysait les difficultés auxquelles sont confrontés en France les musées face aux restes humains.

\section{INDEX}

Mots-clés : Restes humains

\section{AUTEUR}

\section{LAURE CADOT}

Restauratrice d'objets ethnographiques spécialisée dans les matériaux organiques et les restes humains et consultante en conservation préventive.

laure_cadot@yahoo.fr 